\title{
Estimation of Data Transfer Routes Fractal Dimension in Large Scale Networks
}

\author{
Yuriy Danik $^{1}$, Yuriy Kulakov ${ }^{2}$, Vladimir Vorotnikov ${ }^{1}$ and Igor Gumenyuk ${ }^{1}$ \\ 1. Korolyov Zhytomyr Military Institute, Zhytomyr, Ukraine. \\ 2. National technical university of Ukraine "KPI", Kiev, Ukraine.
}

Received: August 24, 2015 / Accepted: September 21, 2015 / Published: January 25, 2016.

\begin{abstract}
The article is devoted to the evaluation of fractal properties of routing data in computer large scale networks. Implemented the study of percolation network topological structures of large dimension and made their transformation into fractal macrostructure. An example of calculating the fractal dimension of the data path for the boundary of the phase transition between the states of network connectivity. The dependence of the fractal dimension of the percolation cluster on the size of the square $\delta$-cover and conductivity value network of large dimension. It is shown that for the value of the fractal dimension of the route $d_{C} \approx 1.5$, network has a stable dynamics of development and size of clusters are optimized with respect to the current load on the network.
\end{abstract}

Key words: large scale network; percolation cluster; fractal dimension; routing

\section{Introduction}

One of the promising areas of modeling complex networks is to use a multifractal dynamics. Currently developed $\mathrm{HN}$ fractal model for solving many problems $[1-3,10,11,16,18]$.

The model of such systems is necessary description of the dynamic behavior of the submitting multifractal curves process. To do this, all the while monitoring is divided into intervals with defined values of linear trend that characterizes the trend in the value of the study. At each interval for the values of the time series is determined by the fractal dimension $d$ and setting-dependence is the slope of the linear friction, and the fractal dimension of the test curves. These models take into account the various parameters that characterize the stability of the systems and processes occurring in them. More often than not, in the construction of fractals involving several structures consisting of different numbers of elements. Moreover,

Corresponding author: Vladimir Vorotnikov, Ph.D. tehn. Sciences / Associate Professor, research field: modeling of complex systems. E-mail: vvvorotnik@ukr.net. the sizes of elements are also different structures [12].

If we imagine a complex network in the form of fractals with cyclically repeating structure, the task mo-modeling their structure reduces to determining the moment phase transition dissipative network topological structures, with the subsequent creation of model objects with fractal structure and the study of their geo-metric characteristics [16].

Analysis of the current scientific literature on the subject suggests that a characteristic to describe the processes occurring in computer networks fields are found on the practice of self-similarity properties of scale invariance or statistical-ray characteristics [15, 17]. These properties are associated with a particular class of physical processes - fractal processes. In this regard, special attention is bought-out design issues constructive methods the Exploration fractality applied to modern computer applications and accounting impact on the character of the formation of control actions with the functioning network of large dimension.

Experimental studies of percolation and fractal structures are complex, not only because of the difficult-not arise in their formation. There are certain 
problems with the localization and determination of structure-round, because they do not possess distinct resonant characteristics.

For example, in [4] deals with the problem of distation clusters of simple sensing nano-satellites, networked. The properties of large random clusters of objects, drawing-forming "a complex network." Introduces the concept of a controlled percolation service area, which is implemented in two phases: the first creates a stochastic basis with a relatively small concentration of nano-satellites, not providing stochastic percolation, and the second best in the intercluster intervals introduces additional nanosatellites for the purpose in obtaining the shortest path percolation through Stochastically formed clusters. In [5] we consider the problem of self-organization in wireless sensor CE's fields and the algorithms to solve it. Estimated time of self-organizing networks using narrowband-gloss and ultra-wideband signals within the standard IEEE 802.15.4. [6] is devoted to the study of complex systems as the network structure. The nodes in such networks represent elements of complex systems, and communication between nodes - the interaction between the elements. In this review are wasps mainly concepts of the theory of complex networks, and outlines the main directions in the study of real set-backed structures. In [7] a new class of event-graphs to model the operation of the network in time. It is assumed that the network monitoring conducted by a "window" method. For the purpose of the anomalies identified, investigated the behavior of normal degree-it, which can be seen in "windows" are considered, my model. The asymptotic of the maximum degree of vertices in the graph, which is generated by the "window" of given size. In [8] proposed a conceptual model of diagnostic infrastructure with intellectual property, which is the core of the administrative services of TCS, providing test, functional diagnostics modules and recovery of workability of the system by data-reconfiguration of the structure of a computer network. In [9] the first, question of reducing the cost of flooding newsletter in dynamic networks through the use of furism "percolation avalanche." -Established relation between the solution in the classical percolation threshold and dynamic cases through integrated statistical characteristics - the average probability of active links.

In the above literature, the definition of fractal properties of the processes carried out only for the systems analysis, as a whole. Questions testing properties of self-similarity-od them subsystems (sub) in other fractal-systems (processes) in the literature are not met.

In this context, the aim of the work is the formalization of the problem and the development of the mathematical apparatus-section of a large dimension network, which has a scale-invariant properties on the clusters, followed by the construction of the data path between nodes and check its fractal properties.

\section{Materials and Methods}

\subsection{The Concept of Complexity Network}

By "complexity networks" refers a system consisting of real objects and relations between them [2]. A complex network graph is modeled, but this graph typically has a specific structure and has the characteristic features. Such networks are called scale-free or scale-invariant (scale-free), as the average degree of a vertex in them is not Xia characteristic, ie, there is no characteristic scale.

For example, for a scale-invariant topology telecommunication network is characterized by a small Number of hubs - the top most - and a large number of vertices of small degree. Complex networks have well defined structure of natural communities: the top of the network are divided into groups that are poorly con-are associated with each other, but have a higher density of edges inside. In this complex network are globally spread with the number of edges $\mathrm{m}$, proportional to the number of vertices n: $m=O(n)$. 
Complex networks are characterized by not only a large number of nodes and the paths between them, and the problem of the Exploration large networks are not limited to, the study of their topology and the properties of each node. Primarily studied in complex networks and their properties aggregate statistical phenomena, in particular:

- statistical characteristics of random structure route in the network;

- path laid down in the networks for the selected criteria of quality;

- nodes (objects)clusters related to defined conditions;

- statistical distributions of nodes, links, clusters and traffic.

\subsection{Percolation Phase Transition in Dissipative Structures}

One of the approaches developed by the study of complexity, networks associated with the model of "flow" at their edges (connections) information flows. Percolation theory solves the problem of the analysis of complex networks in the framework of this mo Delhi. In general, the problem of the flow of information through a complex network of lies well to the formulation of percolation theory [1].

In this formulation, the appearance at a certain concentration - the probability of finding a transit hub in a given region of space stochastic percolation cluster solves the first-approximation with the task - to determine the number of nodes in the cluster.

\subsection{Methods of Determining the Fractal Dimension}

Modern literature uses several basic definitions of fractal dimension: the averaged point wise dimension, correlation dimension and Lyapunov (information) dimension. In most studies, where real-calculated fractal dimensionality, used from 2000 to 20000 points. Direct algorithms used to calculate the fractal dimension to $N_{0}$ points usually contain $N_{0}^{2}$ operations and have high computational complexity. However, the number of operations can be decreased to $N_{0} * \ln \left(N_{0}\right)$ [2].

There are several methods for determining the fractal dimension of the time series [1-3]: First - this is a classic way to the cell when the schedule is served by a series of nets and determine the fractal dimensioning in the same way as for the geometric fractals.

The second method for the investigation of fractal time series was offered by Benoit Mandelbrot and is based on research conducted by the English explorer Hurst and is called R/S method. It is built on the analysis scope parameter (maximum and minimum values in the target segment) and standard deviation [16].

And third is a method based on the change in the length of the curve depending on the scale. If the curve is close to the fractal, then, with downscaling length of the curve will increase in extent.

The fractal dimension, in general, complexity is a measure of the curve. Analyzing the alternation of sections with different fractal dimension and how a system to external and internal factors, we can learn to predict the behavior of the system, diagnose and predict the unstable state. The essential point is the existence of a critical value of the fractal dimension of the time curve, the approach to which the system becomes unstable and goes into an unstable state and parameters quickly either increase or decrease, depending on the trends taking place at this time [3].

Thus, the fractal dimension is defined-term value can be used as an indicator of the crisis, or "flag" disaster. Also, the value of the fractal dimension can serve as an indicator of the number of factors affecting the system.

\subsection{Formulation of Research Problems}

The problem of determining the fractal properties in a complex route network should be split into two stages.

In the first phase by increasing the (saturation) 
nodes in the cluster are determined by the boundary values of the phase transition from the "network is the maximum cluster" in a state of "network consists of multiple clusters-ditch" [2]. This stage characterizes the main feature - the ability to transfer data in a complex network between any two nodes. In the second phase, for the construction of the data path between the complex network nodes determined the fractal dimension of the data path for the phase transition boundary. This step allows you to define quantitative of characteristic data transfer process in a complex network.

\subsection{Determination of the Phase Transition of Network} Connectivity

There is a grid of bonds or matrix, the random part of the cell where the relative $K$ - «white» flow conducting, and the rest - the "black" non-conductive thread. It is necessary to find the minimum concentration of the "white" cells in which is formed a through path for relations or white cells through the matrix in a predetermined direction [1,9].

In other words, you want to find such a concentration of $K_{n}$, where the whole matrix as a whole spends. When pre-achieving conduction properties of the network quality and change abruptly: the image of a safe way (or it is destroyed), there is (or attenuated) epidemic, is recreated destroyed social network, there is a traffic jam in traffic, and so on. $N$. The concentration of $K$ - the proportion of white nodes at random uniform filling of the lattice or matrix - is the probability of the existence of "white" of the object in the cell matrix $[1,3,6]$.

The calculation of the fractal dimensionality of the percolation clusterisation in practice on the basis of the correlation dimension:

$$
d_{c}=\lim _{\delta \rightarrow 0} \frac{\ln C(\delta)}{\ln \delta}
$$

where $C(\delta)$ - correlation function.

$C(\delta)$ is calculated, as a rule, like the ratio of points $\mathrm{n}$ numbers, pair wise distances between those smaller by the $\delta$ to square of the total number of $N$ points.

$$
C(\delta)=\frac{n}{N^{2}}
$$

$\delta$ - the size of the geometric structure, which is covered by a set of points.

Distance between points $P_{1}\left(x_{1}, y_{1}\right)$ и $P_{2}\left(x_{2}, y_{2}\right)$ is defined, generally, as

$$
r=\sqrt{\left(x_{2}-x_{1}\right)^{2}+\left(y_{2}-y_{1}\right)^{2}}
$$

Therefore, it is necessary to calculate the number of points $n$, the distance between them does not exceed $r \leq \delta$, a $\delta$-is given by.

\subsection{The Definitions of the Fractal Dimension of the} Route in the Large Dimension Network

Suppose we have a set of $G$ set in Euclidean space of dimension $d$. This set of cubes covered by $d$ dimension, wherein each cube edge length exceeds a certain $\delta$ value, i.e. $\delta_{i}<\delta$. Introduced depending on a parameter $d$ and $\delta$ the sum of all the elements of cover:

$$
l_{d}(\delta)=\sum_{i} \delta_{i}^{d}
$$

The lower limit of this sum:

$$
L_{d}(\delta)=\inf \sum_{i, \delta_{i}<\delta} \delta_{i}^{\tau}
$$

When reducing the maximum $\delta$ length if the $d$ parameter will be sufficiently large, then will be carried out:

$$
\lim _{\delta \rightarrow 0} L_{d}(\delta) \rightarrow 0
$$

For some sufficiently small value of the parameter $d$ will be carried out:

$$
\lim _{\delta \rightarrow 0} L_{d}(\delta) \rightarrow 0
$$

An intermediate, the critical value $d_{x}$, for which:

$$
\lim _{\delta \rightarrow 0} L_{d}(\delta)=\left\{\begin{array}{l}
0, d>d_{x} \\
\infty, d<d_{x}
\end{array}\right.
$$

is the dimension of Hausdorff-Besicovitch (or fractal dimension). 
Despite the fact that the dimension of Hausdorff-Besicovitch from a theoretical point of view is defined flawlessly for real fractal objects calculation of this dimension is very difficult. In this introduces several simple indicator - capacity dimension $d_{c}$. In determining this amount, use of squares with edges of the same size. In this case, fair:

$$
L_{d}(\delta)=N(\delta) \delta^{d_{c}}
$$

where $N(\delta)$ - the number of blocks, covers an $G$ area.

By taking the logarithm and passing to the limit with a decrease of the cube, covers an area $G$. By taking the logarithm and passing to the limit with a decrease of the cube $\delta \rightarrow 0$, we obtain:

$$
d_{c}=-\lim _{\delta \rightarrow 0} \frac{\log N(\delta)}{\log \delta}
$$

if this limit exists.

For regular self-similar fractal capacity dimension and the dimension of Hausdorff-Besicovitch owls, fall, so they are often not terminological differences, and are just talking about the fractal dimension of the object $[4,6]$.

\section{Results and Discussion}

\subsection{Example of Calculating the Fractal Dimension of the Route in a Complex Computer Network}

Given a complex mesh network. Them-ting shape and size of the cell topology real network is not required. The model simulates the connection nodes objects of the network: network entities connected if contacted with sides of square cells in matrix in which they are located. Under these conditions, the growth of concentration of "white" are formed and grow clusters con-associated "white" objects - nodes.

To determine the phase transition in a scale-invariant network calculate the fractal dimension of the set of the plurality of nodes using the following algorithm [1]:

- Generate a sequence of evenly distributed variables.
- Find the center of mass of the cluster. In the two-dimensional case coordinates of the center of mass are given by

$$
x_{c}=\frac{1}{n} \sum_{i=1}^{n} x_{i} y_{c}=\frac{1}{n} \sum_{i=1}^{n} y_{i}
$$

where $n$ - number of nodes in the cluster, $\left(x_{i}, y_{i}\right)-$ $i$-th node coordinates.

Find the number of nodes in the cluster $m(L)$, inside a square with sides $L$ and center of the center of mass of the cluster $\left(x_{c}, y_{c}\right)$.

Increase the amount of $L$ twice and repeat the $\mathrm{p}$. 3-4 $s$ times, while the square will not cover the entire cluster.

Building a relationship $\operatorname{Ln}(m)$ from $\operatorname{Ln}(L)$.

Calculate the fractal dimension of least squares [1]

$$
d_{f}=B=\frac{s \sum_{i=1}^{s} X_{i} Y_{i}-\sum_{i=1}^{s} X_{i} * \sum_{i=1}^{s} Y_{i}}{\Delta}
$$

$$
\begin{gathered}
\delta d_{f}=\delta B=\frac{\sigma_{B}}{\sqrt{s}}, \text { where } X_{i}=\ln L_{i}, Y_{i}=\ln m\left(L_{i}\right) \\
A=\frac{\sum_{i=1}^{s} X_{i}^{2} \sum_{i=1}^{s} Y_{i}-\sum_{i=1}^{s} X_{i} * \sum_{i=1}^{s} X_{i} Y_{i}}{\Delta} \\
\sigma_{B}^{2}=\frac{s \sigma_{y}^{2}}{\Delta}, \quad \sigma_{Y}^{2}=\frac{1}{s} \sum_{i=1}^{s}\left(Y_{i}-A-B X_{i}\right)^{2} \\
\Delta=s \sum_{i=1}^{s} X_{i}^{2}-\left(\sum_{i=1}^{s} X_{i}\right)^{2}
\end{gathered}
$$

Repeat 1-5 $n$ times with different sequences of random numbers. Find the average value of the fractal dimension.

Fig. 1 shows the variations of saturation of cluster with nodes of the network. The total number of units of a complex network crashed, been spent on clusters. Fig. 1a network is divided into 400 clusters, while there are empty cells (clusters do not contain nodes). $1 \mathrm{~b}-1 \mathrm{c}$ on the cluster size is taken away, at. Fig. 1d network is divided into 4 clusters, nodes are filled evenly.

We are interested in the behavior of a complex network for critical $\ln (L) \cong 5 \ldots 9$. Precisely in this 


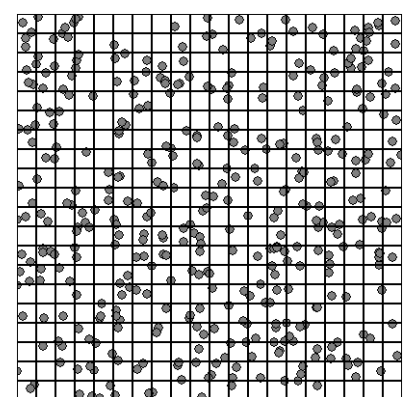

(a)

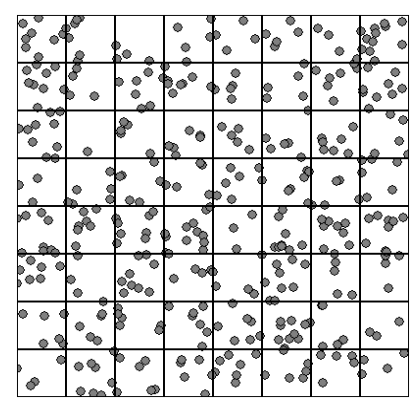

(b)

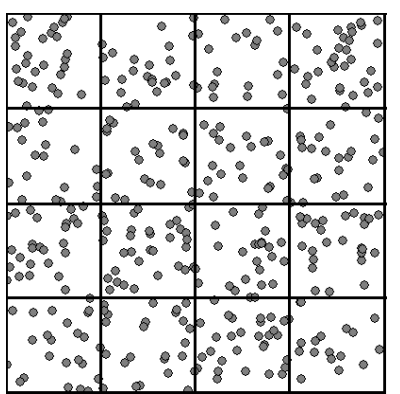

(c)

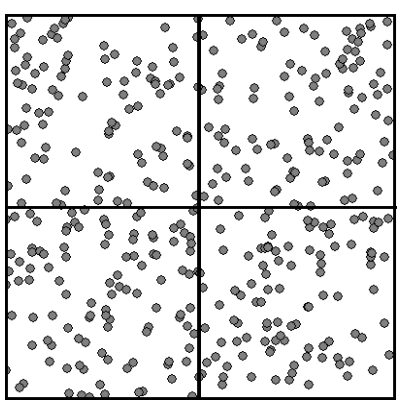

(d)

Fig. 1 A complex network consists of: a) 400 clusters; b) 64 clusters; c) 16 clusters; d) 4 clusters.

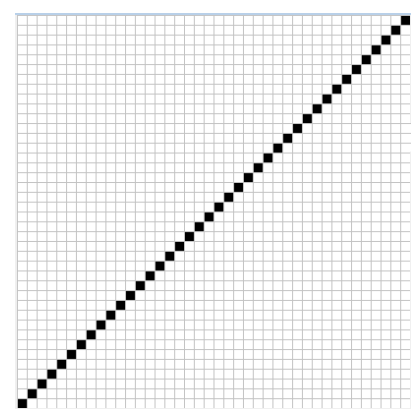

(a)

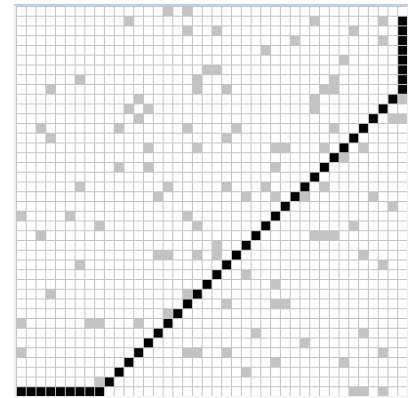

(b)

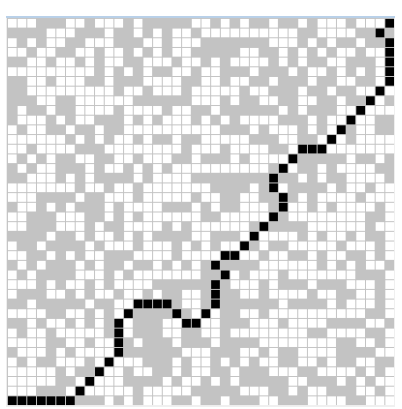

(c)

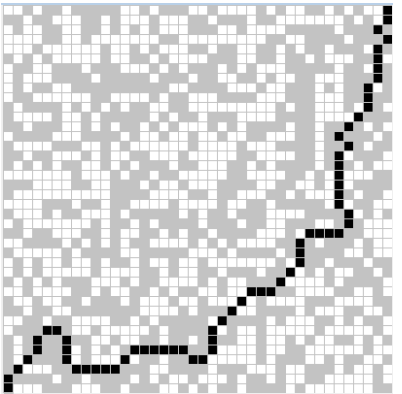

(d)

Fig. 2 Changing cross of a complex network, depending on the number of available cluster nodesSimulation of the process of the cluster node saturation, by analogy with the concept of "penetrability" in percolation theory, corresponds to the process state changes the network in which the nodes within the cell are reachable (network is connected). Conversely, when withdrawn, the number of non-conductive black cells in a complex system is based on a pro-phase transition from the conduction state when the network consists of a single global cluster connected to the state of nonconduction when the network-decay is a few clusters. Fig. 3 shows the number of nodes (cell) referred to the cluster of scale 1.

point the network abruptly changes its conductivity properties. In this determination step percolation cluster ends. The outcome will be the definition of the boundary values of the cluster size on the network, in which a complex network retains its conductivity.

The second step analyzes the geometrical shape data path between two nodes A and B. From Fig. 3 shows that as the number of inaccessible nodes in the network, the shape of the data path between nodes A and $B$ changes from linear to nonlinear form. The more inaccessible nodes in the network the "confusing" becomes a way of passing data between nodes. Route information flow in a complex network between A and B (Fig. 4) has the shape of "coastline" $[3,4]$. To check the properties of such a route in the network determine its capacity dimension [2].

Suppose at some point had to use the fractal coating $N(\delta)$ squares with edges size $\delta$, and another -
$N\left(\delta^{\prime}\right)$ elements with the parties $\delta^{\prime}$. In view of the proposed power dependence is true:

$$
N(\delta) \cong \frac{1}{\delta^{d_{c}}}, \quad N\left(\delta^{\prime}\right) \cong \frac{1}{\delta^{d_{c}}} .
$$

where the value of $d_{c}\left(\delta / \delta^{\prime}\right)$ can be estimated as:

$$
d_{c}=-\frac{\log \left(N(\delta) / N\left(\delta^{\prime}\right)\right)}{\log \left(\delta / \delta^{\prime}\right)}
$$

According to (1-3), the route covered $\delta$ - coated with a side of the square $\delta=3,4,5,6$. Dependence of the fractal dimension of the route from the rate of attitude change $\delta / \delta^{\prime}(11)$ shown in Fig. 5.

Analysis of the results allows us to define the range of variation-casting ratio of the lengths of the squares in the fractal coating that maintains the properties of Ca-similarity. This allows, for known data-intensity loaded the network, identify parts of the route on which observed overload and predict their length-productivity of. 


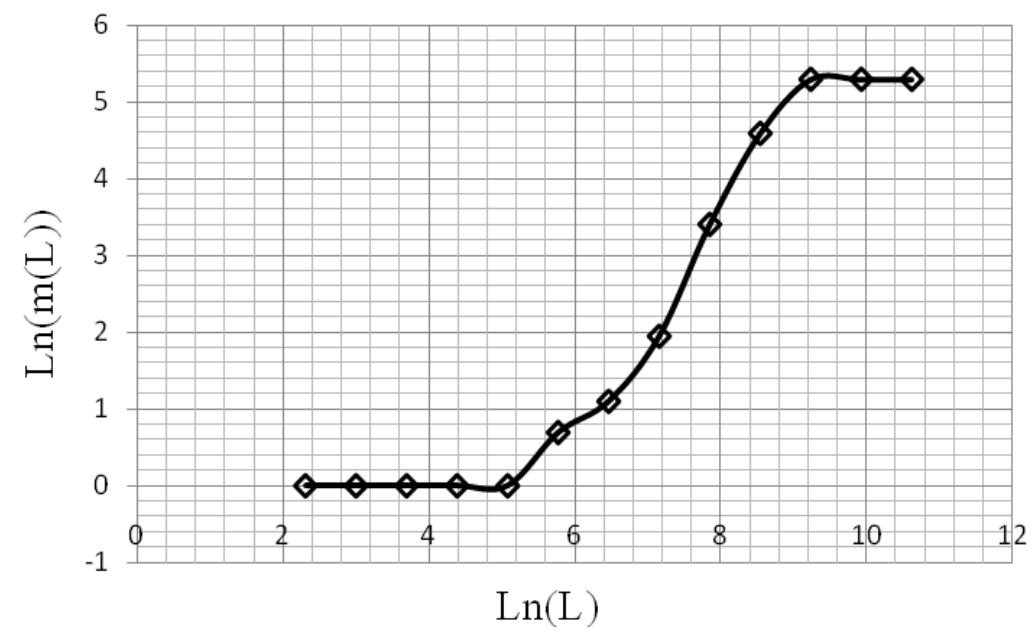

Fig. 3 Phase transition: dependence of the number of nodes included in the cluster to scale $L$

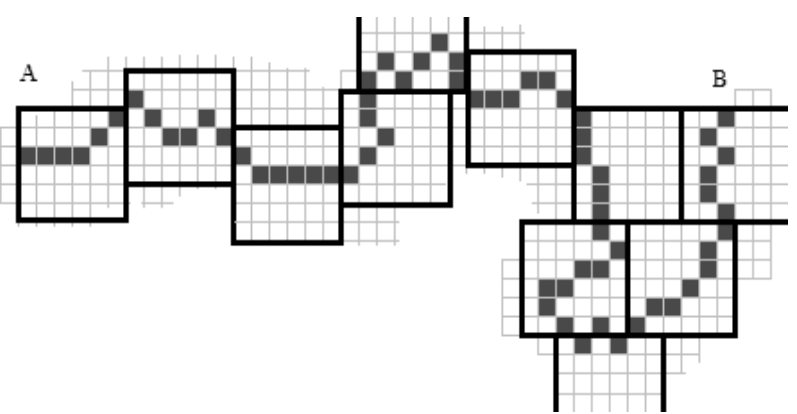

(a)

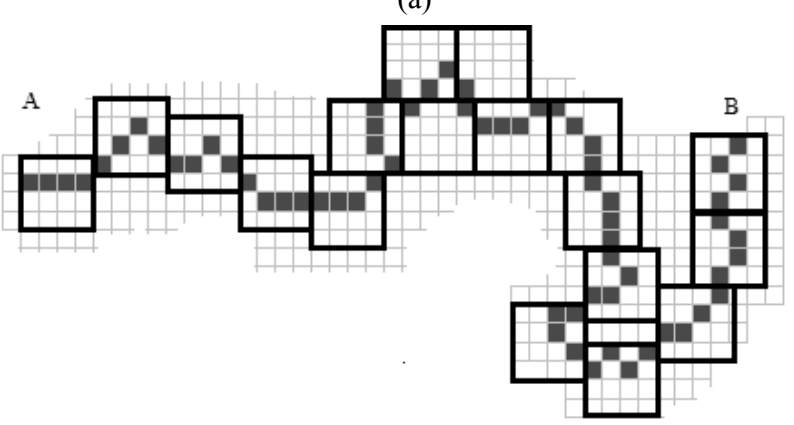

(c)

Fig. 4 Cover the data path squares with sides:

(a) $\delta=6$; (b) $\delta=5$; (c) $\delta=4$; (d) $\delta=3$

Also, one of the possible areas of practical application of the results-ray may be the use of this information by partitioning the nodes included in the route on the clusters.

\section{Conclusions}

Interpretation of the results can be as follows:

The objective function is network connectivity between any two nodes.

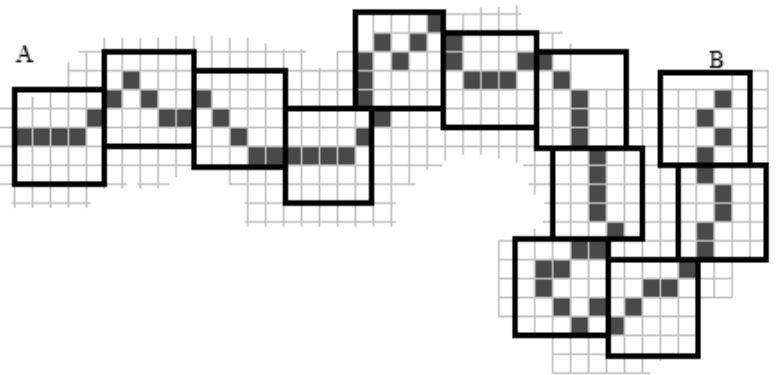

(b)

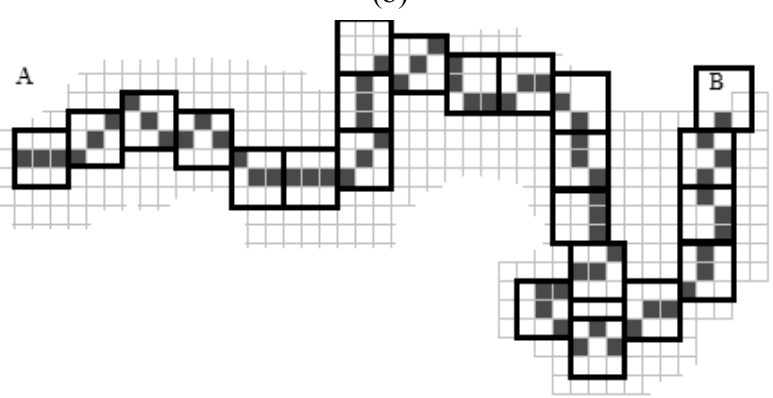

(d)

The network can be in two states: the conduction and no conduction. To determine these conditions network is divided into clusters, the number of which is specified by saturation of nodes in a certain area.

At the percolation cluster size and a large network of which describes the operation of the network in limited mode, showing the properties of geometric fractal with decreasing cluster size. By analyzing the 


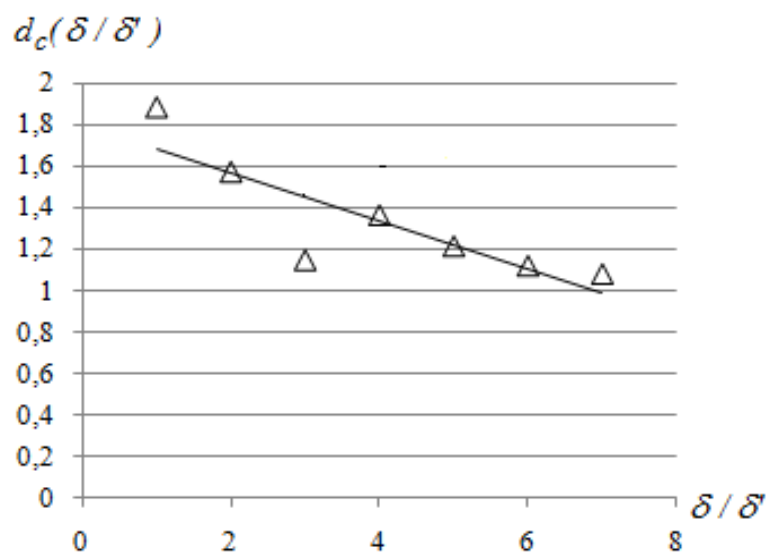

Fig. 5 The dependence of the fractal dimension of the value $\delta / \delta^{\prime}, \delta^{\prime}=\delta-i, i=1 . . \delta$

alternating sections with different fractal time-dimensionality and how to network to external and internal factors that can predict the behavior of the network, diagnose and predict its unstable state.

External factor in this case is intensity of traffic determines the amount of black cells in the network model, through which no data transmission capabilities.

Internal factor is the possibility of reorganizations of network - change cluster size.

Thus, when the fractal dimension of less than 1.4, a major influence on the development of the network has the dimension of the cluster-ditch - in such clusters in the presence of conductive network (traffic intensity) remains the property of connectedness, and the structure of the network is redundant; if the size-of about 1.5, the external and internal factors corresponding to the network in different directions, but more or less cancel each other out; if the fractal dimension of a much-1.6, the system becomes non-stable and ready to move to a new state.

\section{References}

[1] Ju. Ju. Tarasevich, Perkoljacija: teorija, prilozhenija, algoritmy. M.: URSS, (2002), 109 s.

[2] D. V. Landje, A. A. Snarskij, I. V. Bezsudnov. Internetika: Navigacija $\mathrm{v}$ slozhnyh setjah: modeli i algoritmy. M.: Knizhnyj dom «Liberkom», (2009), 264 s.

[3] A.G. Dodonov, D.V. Landje. Zhivuchest' informacionnyh system. Kiev: Naukova dumka, (2011), 256 s.
[4] Ja.A. Mostovoj. Dvuhfaznye operacii v bol'shih setjah nanosputnikov. Komp'juternaja optika, (2013), tom 37, №1, s. 129-139.

[5] V. Ju. Jurkin, T. I. Mohseni. Ierarhicheskie podhody k samoorganizacii $\mathrm{v}$ besprovodnyh sverhshirokopolosnyh sensornyh setjah na osnove haoticheskih radioimpul'sov. TRUDY MFTI, (2012), tom 4, № 3, s.151-161.

[6] I. A. Evin. Vvedenie v teoriju slozhnyh setej. Komp'juternye issledovanija i modelirovanie, (2010), tom 2, № 2, s. 121-141.

[7] A. A. Grusho, E. E. Timonina Model' sluchajnyh grafov dlja opisanija vzaimodejstvij $\mathrm{v}$ seti. Informatika $\mathrm{i}$ ee primenenija, (2012), tom 6, № 4, s. 57-60.

[8] S. G. Karpenko, M. A. Kovalenko, M. A. Miroshnik, S. V. Panchenkoю. Podhod k proektirovaniju komp'juternyh setej $\mathrm{s}$ intellektual'noj diagnosticheskoj infrastrukturoj. ITKS, (2011), №6, s. 51-59.

[9] A. S. Golubev, M. Ju. Zvjagin, D. S. Milovanov. Jeffekt perkoljacii $\mathrm{v}$ informacionnyh setjah $\mathrm{s}$ neustojchivymi svjazjami. Informacionnye tehnologii. Vestnik Nizhegorodskogo universiteta im. N.I. Lobachevskogo, (2011), № 3 (2), s. 260-263.

[10] R. Albert, A.-L. Barabasi, Rev. Mod. Phys. 74 (2002) 47.

[11] J.F.F. Mendes, S.N. Dorogovtsev, A.F. IoNe, Evolution ofNetworks: From Biological Nets to the Internet and WWW, Oxford University Press, Oxford, 2003.

[12] R. Cohen, S. Havlin. Fractal dimensions of percolating networks / Physica A 336 (2004) Pp. 6- 13

[13] Zhang, Yu. The fractal volume of the two-dimensional invasion percolation cluster. Comm. Math. Phys. 167 (1995), no. 2, 237-254. http://projecteuclid.org/ euclid.cmp/1104271992.

[14] Shu Tanaka, Ryo Tamura. Network-Growth Rule Dependence of Fractal Dimension of Percolation Cluster on Square Lattice Journal of the Physical Society of Japan, May 15, 2013, Vol. 82, No. 5. (doi: 10.7566/ JPSJ. 82.053002)

[15] P. Clerca, G. Girauda, J.M. Laugierab \& J.M. Luckc The electrical conductivity of binary disordered systems, percolation clusters, fractals and related models Advances in PhysicsVolume 39, Issue 3, (1990). Pp 191-309.

[16] Evertsz, C.J.G. and B.B. Mandelbrot, Multifractal measures. in Fractals and Chaos, edited by Peitgen, H-O., H. Jurgens, and D. Saupe, Springer-Verlag, New York. (1992), 921-969.

[17] Berkowitz, B. and Braester, C. Dispersion in subrepresentative elementary volume fracture networks: Percolation theory and random walk approaches, Water Resources Research 27, (1991), 3159-3164.

[18] Reuven Cohen and Shlomo Halvin. Fractal dimensions of percolating networks/ Physica A: Statistical Mechanics and its Applications, (2004) 6-13. 